\title{
Bifurcation of Nongeneric Homoclinic Orbit Accompanied by Pitchfork Bifurcation
}

\author{
Fengjie Geng and Song Li \\ School of Science, China University of Geosciences (Beijing), Beijing 100083, China \\ Correspondence should be addressed to Fengjie Geng; gengfengjie@cugb.edu.cn
}

Received 15 February 2014; Accepted 11 March 2014; Published 7 April 2014

Academic Editor: Yonghui Xia

Copyright (C) 2014 F. Geng and S. Li. This is an open access article distributed under the Creative Commons Attribution License, which permits unrestricted use, distribution, and reproduction in any medium, provided the original work is properly cited.

The bifurcation of a nongeneric homoclinic orbit (i.e., the orbit comes from the equilibrium along the unstable manifold instead of the center manifold) connecting a nonhyperbolic equilibrium is investigated, and the nonhyperbolic equilibrium undergoes a pitchfork bifurcation. The existence (resp., nonexistence) of a homoclinic orbit and an 1-periodic orbit are established when the pitchfork bifurcation does not happen, while as the nonhyperbolic equilibrium undergoes a pitchfork bifurcation, we obtain the sufficient conditions for the existence of homoclinic orbit and two or three heteroclinic orbits, and so forth. Moreover, we explore the difference between the bifurcation of the nongeneric homoclinic orbit and the generic one.

\section{Introduction}

It is well known that the nonhyperbolic equilibrium is unstable and always undergoes a saddle-node (resp., transcritical or pitchfork) bifurcation. So the bifurcation problems of homoclinic or heteroclinic orbits with nonhyperbolic equilibria are more difficult and challenging. And few of the papers take into account the homoclinic or heteroclinic orbits with nonhyperbolic equilibria. Zhu [1] gave the sufficient conditions for the existence of nongeneric heteroclinic orbits accompanied with saddle-node bifurcation by extending exponential trichotomy. Klaus and Knobloch [2] discussed the bifurcation of homoclinic orbit to a saddle-center in reversible system. Liu et al. [3] considered the bifurcations of homoclinic orbit with a nonhyperbolic equilibrium for a high dimensional system; they achieved the persistence of homoclinic orbit and the bifurcation of periodic orbit for the system accompanied by a pitchfork bifurcation. In 2012, we discussed the bifurcations of generic heteroclinic loop accompanied by pitchfork bifurcation [4]. For other works about bifurcations of the homoclinic or heteroclinic orbits with nonhyperbolic equilibria, the readers may see [5-8] and references therein.

Inspired by the above works, we deal with the nongeneric homoclinic bifurcation accompanied by a pitchfork bifurcation in a 4-dimensional system. By extending the method established in [7], we give the sufficient conditions for the existence of a generic (resp., a nongeneric homoclinic) orbit and a periodic orbit when pitchfork bifurcation does not happen, while the nonhyperbolic equilibrium undergoes a pitchfork bifurcation, we achieve the existence of homoclinic orbits connecting the bifurcated equilibrium and three heteroclinic orbits, where we may know the difference of bifurcations between the nongeneric homoclinic orbit and the generic one.

The rest of the paper is organized as follows. In Section 2, we present some hypotheses and give the normal form for the system considered in this paper. The Poincaré map and successor function are achieved in Section 3. Finally, the existence and nonexistence of homoclinic, heteroclinic, and periodic orbits are given in Section 4.

\section{Hypotheses and Normal Form}

Consider the following $C^{r}(r \geq 3)$ system:

$$
\dot{w}=H(w, \lambda, \mu),
$$

and its unperturbed system

$$
\dot{w}=F(w),
$$

where $w \in \mathbb{R}^{4}, \lambda \in \mathbb{R}, \mu \in \mathbb{R}^{l}(l \geq 3)$, and $0 \leq \lambda,|\mu| \ll 1$, $G(w, 0,0)=F(w), H(O, 0, \mu)=0$; namely, the origin is an equilibrium of system (2). 
Assume system (2) has a homoclinic orbit $\Gamma$ connecting the origin with $\gamma( \pm \infty)=O$; denote $\Gamma=\{w=\gamma(t): t \in \mathbb{R}\}$. Moreover, the linearization $D F(O)$ has four real eigenvalues $0, \lambda_{1},-\rho_{1}$, and $-\rho_{2}$ satisfying $-\rho_{2}<-\rho_{1}<0<\lambda_{1}$. Obviously, the nonhyperbolic equilibrium $O$ has a 2-dimensional stable manifold $W^{s}$, an 1- dimensional center manifold $W^{c}$, and 1dimensional unstable manifold $W^{u}$.

The following hypotheses will be needed in the whole paper:

$\left(\mathrm{H}_{1}\right) \operatorname{dim}\left(T_{\gamma(t)} W^{c u} \cap T_{\gamma(t)} W^{s}\right)=\operatorname{dim}\left(T_{\gamma(t)} W^{u} \cap\right.$ $\left.T_{\gamma(t)} W^{s}\right)=1$, which means that the homoclinic orbit is nongeneric, and the orbit is generic if it comes from the origin along the center manifold; the bifurcation for generic homoclinic orbit one may see [3]:

$\left(\mathrm{H}_{2}\right)$

$$
\begin{aligned}
& \lim _{t \rightarrow+\infty} T_{\gamma(t)} W^{c u}=T_{O} W^{u}+e^{+} \\
& \lim _{t \rightarrow-\infty} T_{\gamma(t)} W^{s}=T_{O} W^{s s}+e^{-}
\end{aligned}
$$

where $W^{s s}$ denotes the strong stable manifold of $O$, $e^{ \pm}=\lim _{t \rightarrow \mp \infty}=\dot{\gamma}(t) /|\dot{\gamma}(t)|$.

$\left(\mathrm{H}_{3}\right)$ Let $x$-axis be the tangent space of the center manifold at $O$, and let $\theta(x, \lambda, \mu)$ be the vector field defined on the center manifold and satisfies

$$
\begin{gathered}
\theta(0, \lambda, \mu)=0, \quad \frac{\partial \theta}{\partial x}(0,0,0)=0, \quad \frac{\partial^{2} \theta}{\partial x^{2}}(0, \lambda, \mu)=0 \\
\frac{\partial^{3} \theta}{\partial x^{3}}(0,0,0)>0, \quad \frac{\partial^{2} \theta}{\partial x \partial \lambda}(0,0,0)<0 \\
\frac{\partial^{2} \theta}{\partial x \partial \mu}(0,0,0)=0 .
\end{gathered}
$$

According to Wiggins [9], under the above assumption, the origin is a pitchfork bifurcation point, and $\lambda$ is the parameter controlling the pitchfork bifurcation; that is to say, under small perturbation when $\lambda>0$ the origin is perturbed into three hyperbolic saddles $\mathrm{O}^{0}, \mathrm{O}^{+}, \mathrm{O}^{-}$(one may see Figure 1). Denote $O^{0}=O=(0,0,0,0)^{\prime}, O^{+}=O^{0}+\left(\sqrt{\lambda_{p}}, 0,0,0\right)^{\prime}$, and $O^{-}=O^{0}+\left(-\sqrt{\lambda_{p}}, 0,0,0\right)^{\prime}$, where $\lambda_{p}=\theta_{0} \lambda+O\left(\lambda^{2}\right)+O(\lambda \mu)$, $\theta_{0}=-\left(\partial^{2} \theta / \partial x \partial \lambda\right)(0,0,0) /\left(\partial^{3} \theta / \partial x^{3}\right)(0,0,0)$. In the whole paper, the sign " $/$ " denotes the transpose of the vector. It is easy to see that $\operatorname{dim}\left(W_{\mathrm{O}^{+}}^{u}\right)=\operatorname{dim}\left(W_{\mathrm{O}^{-}}^{u}\right)=3, \operatorname{dim}\left(W_{\mathrm{O}^{+}}^{s}\right)=$ $\operatorname{dim}\left(W_{\mathrm{O}^{-}}^{s}\right)=1, \operatorname{dim}\left(W_{\mathrm{O}^{0}}^{s}\right)=\operatorname{dim}\left(W_{\mathrm{O}^{0}}^{u}\right)=2$.

According to the invariance of the manifolds, we may introduce a scale transformation and straighten the local manifolds of $W^{c}, W^{u}, W^{s s}, W^{s}$; then system (2) has the following expression in the small neighborhood $U$ of the origin:

$$
\begin{aligned}
\dot{x} & =-\lambda_{p} x+x^{3}+\mu h\left(x, y_{1}, u, y_{2}\right)+\text { h.o.t., } \\
\dot{y}_{1} & =\left[-\rho_{1}(\alpha)+\ldots\right] y_{1}+O\left(y_{2}\right)[O(x)+O(u)], \\
\dot{u} & =\left[\lambda_{1}(\alpha)+\ldots\right] u, \\
\dot{y}_{2} & =\left[-\rho_{2}(\alpha)+\ldots\right] y_{2}+O\left(y_{1}\right)\left[O(x)+O\left(y_{1}\right)+O(u)\right],
\end{aligned}
$$

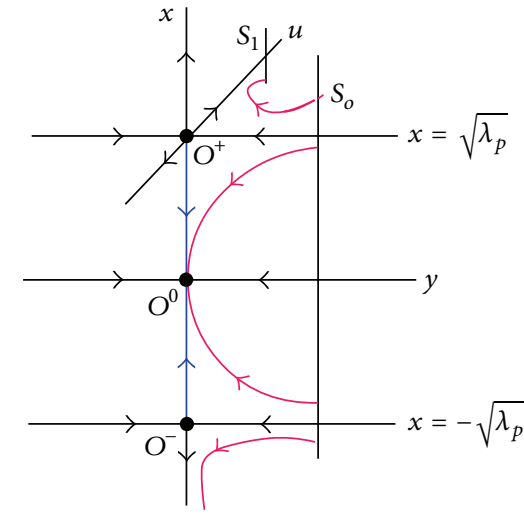

FIgURE 1: The orbits near nonhyperbolic equilibrium.

Due to the normal form (5) and $\left(\mathrm{H}_{1}\right)$, we may choose $T$ $(T \gg 1)$ such that $\gamma(-T)=(0,0, \delta, 0)^{\prime}, \gamma(T)=\left(0, \delta, 0, \delta_{y}\right)^{\prime}$, where $\delta>0$ is small enough such that $\left\{\left(x, y_{1}, u, y_{2}\right)\right.$ : $\left.|x|,\left|y_{1}\right|,|u|,\left|y_{2}\right|<2 \delta\right\} \subset U$. Obviously, $\left|\delta_{y}\right|=o(\delta)$.

Take into account the linear variational system:

$$
\dot{U}=A U
$$

and its adjoint system

$$
\dot{\Phi}=-A^{\prime} \Phi
$$

where $A=D F(\gamma(t))$ and $A^{\prime}$ is the transpose of $A$.

We introduce the following lemma; it is very significant in this paper.

Lemma 1. Let $U(t)=\left(u_{1}(t), u_{2}(t), u_{3}(t), u_{4}(t)\right)$ be a fundamental solution matrix of system (6), we can select $u_{1}(t) \epsilon$ $\left(T_{\gamma(t)} W^{c u}\right)^{c} \bigcap\left(T_{\gamma(t)} W^{s}\right)^{c}, \quad u_{2}(t)=\dot{\gamma}(t) /|\dot{\gamma}(-T)| \in T_{\gamma(t)}$ $W^{u} \cap T_{\gamma(t)} W^{s}, u_{3}(t) \in T_{\gamma(t)} W^{c u} \bigcap\left(T_{\gamma(t)} W^{s}\right)^{c}, u_{4}(t) \in\left(T_{\gamma(t)}\right.$ $\left.W^{c u}\right)^{c} \cap T_{\gamma(t)} W^{s}$ such that

$$
\begin{aligned}
& U(-T)=\left(\begin{array}{llll}
u_{11} & 0 & 1 & u_{41} \\
u_{12} & 0 & 0 & u_{42} \\
u_{13} & 1 & 0 & u_{43} \\
0 & 0 & 0 & u_{44}
\end{array}\right), \\
& U(T)=\left(\begin{array}{cccc}
1 & 0 & u_{31} & 0 \\
0 & u_{22} & u_{32} & 0 \\
0 & 0 & u_{33} & 0 \\
u_{14} & u_{24} & u_{34} & 1
\end{array}\right),
\end{aligned}
$$

where $u_{12} \neq 0, u_{i i} \neq 0$ for $i=2,3,4$, and $\left|u_{1 j} u_{12}^{-1}\right| \ll 1$ for $j=$ $1,3,\left|u_{3 j} u_{33}^{-1}\right| \ll 1$ for $j=1,2,4$, and $\left|u_{4 j} u_{44}^{-1}\right| \ll 1$ for $j=$ $1,2,3$.

Proof. According to the hypotheses $\left(\mathrm{H}_{1}\right)$ and $\left(\mathrm{H}_{2}\right)$, one may easily obtain the existence of the $u_{2}(t), u_{3}(t)$, and $u_{4}(t)$ with the given expression at $t= \pm T$. Based on the condition $\left(\mathrm{H}_{2}\right)$, we take $\bar{u}_{1}(t) \in\left(T_{\gamma(t)} W^{c u}\right)^{c} \bigcap\left(T_{\gamma(t)} W^{s}\right)^{c}$, satisfying

$$
\bar{u}_{1}(-T)=\left(\bar{u}_{11}, \bar{u}_{12}, \bar{u}_{13}, \bar{u}_{14}\right)^{\prime}, \quad \bar{u}_{1}(T)=(1,0,0,0)^{\prime} .
$$

where $\alpha=(\lambda, \mu), \lambda_{1}(0)=\lambda_{1}, \rho_{i}(0)=\rho_{i}$, for $i=1,2$. 
Let $u_{1}(t)=\bar{u}_{1}(t)+u_{4}(t) u_{14}$, where $u_{14}=-u_{44}^{-1} \bar{u}_{14}$, so we have $u_{1}(t) \in\left(T_{\gamma(t)} W^{c u}\right)^{c} \bigcap\left(T_{\gamma(t)} W^{s}\right)^{c}$, satisfying

$$
u_{1}(-T)=\left(u_{11}, u_{12}, u_{13}, 0\right)^{\prime}, \quad u_{1}(T)=\left(1,0,0, u_{14}\right)^{\prime},
$$

where $u_{1 j}=\bar{u}_{1 j}-u_{4 j} u_{44}^{-1} \bar{u}_{14}, j=1,2,3$, obviously $u_{12} \neq 0$.

Noticing that the strong inclination property holds, it then follows that $u_{33} \neq 0, u_{44} \neq 0$. By $\operatorname{diag} A(t) \rightarrow \operatorname{diag}\left(0,-\rho_{1}\right.$, $\left.\lambda_{1},-\rho_{2}\right)$ as $|t| \rightarrow \infty$, one can easily know that $\left|u_{1 j} u_{12}^{-1}\right| \ll 1$ for $j=1,3,\left|u_{3 j} u_{33}^{-1}\right| \ll 1$ for $j=1,2,4$, and $\left|u_{4 j} u_{44}^{-1}\right| \ll 1$ for $j=1,2,3$.

The proof is then finished.

Let $\Phi(t)=\left(\phi_{1}, \phi_{2}, \phi_{3}, \phi_{4}\right)=\left(U^{-1}(t)\right)^{\prime}$; from the matrix theory, we know that $\Phi(t)$ is the fundamental solution matrix of (7).

Introduce the following local moving frame coordinates:

$$
e(t)=\gamma(t)+U(t) L(t)
$$

where $L(t)=\left(l_{1}(t), 0, l_{3}(t), l_{4}(t)\right)^{\prime}$. Define the cross sections:

$$
\begin{aligned}
& S_{0}=\left\{w=e(T)=(x, y, u):\left|l_{i}\right|<\delta\right\} \subset U, \\
& S_{1}=\left\{w=e(-T)=(x, y, u):\left|l_{i}\right|<\delta\right\} \subset U, \quad i=1,2 .
\end{aligned}
$$

Notice that if $q_{0} \in S_{0}, q_{1} \in S_{1}$, then

$$
\begin{array}{r}
q_{0}=\left(x_{0}, y_{10}, u_{0}, y_{20}\right)^{\prime}=\gamma(T)+U(T) L(T), \\
L(T)=\left(l_{10}, 0, l_{30}, l_{40}\right)^{\prime}, \\
q_{1}=\left(x_{1}, y_{11}, u_{1}, y_{21}\right)^{\prime}=\gamma(-T)+U(-T) L(-T), \\
L(-T)=\left(l_{11}, 0, l_{31}, l_{41}\right)^{\prime} .
\end{array}
$$

We may easily obtain the new coordinates for $q_{0}$ and $q_{1}$ as follows:

$$
\begin{aligned}
l_{10}= & \left(u_{12}\right)^{-1} y_{11}-u_{42}\left(u_{12} u_{44}\right)^{-1} y_{21}, \\
l_{30}= & x_{1}-u_{11}\left(u_{12}\right)^{-1} y_{11} \\
& +\left(u_{11}\left(u_{12}\right)^{-1} u_{42}-u_{41}\right)\left(u_{44}\right)^{-1} y_{21}, \\
l_{40}= & \left(u_{44}\right)^{-1} y_{21}, \\
y_{10} \approx & \delta \\
l_{11}= & x_{0}-u_{31}\left(u_{33}\right)^{-1} u_{0}, \\
l_{31}= & \left(u_{33}\right)^{-1} u_{0}, \\
l_{41}= & y_{20}-\delta_{y}-u_{14} x_{0} \\
& +\left(u_{14} u_{31}-u_{34}\right)\left(u_{33}\right)^{-1} u_{0}, \\
u_{1} \approx & \delta .
\end{aligned}
$$

\section{Poincaré Map and Successor Function}

In this section, we establish the Poincare map in the new coordinate system and then derive the successor function.

(1) Establishment of the map $P_{1}: S_{1} \mapsto S_{0}$.
Putting $e(t)=\gamma(t)+U(t) L(t)$ into system (1), we have

$$
\begin{aligned}
\dot{\gamma}(t)+ & \dot{U}(t) L(t)+U(t) \dot{L}(t) \\
= & H(\gamma(t)+U(t) L(t), \lambda, \mu) \\
= & H(\gamma(t), 0,0)+H_{\gamma}(\gamma(t), 0,0) U(t) L(t) \\
& +H_{\lambda}(\gamma(t), 0,0) \lambda+H_{\mu}(\gamma(t), 0,0) \mu+\text { h.o.t. } \\
= & F(\gamma(t))+D F(\gamma(t)) U(t) L(t) \\
& +H_{\lambda}(\gamma(t), 0,0) \lambda+H_{\mu}(\gamma(t), 0,0) \mu+\text { h.o.t.. }
\end{aligned}
$$

Since $\dot{\gamma}(t)=F(\gamma(t))$ and $\dot{U}(t)=A U(t)=D F(\gamma(t)) U(t)$, it then follows that

$$
\dot{L}(t)=U^{-1}(t)\left[H_{\lambda}(\gamma(t), 0,0) \lambda+H_{\mu}(\gamma(t), 0,0) \mu\right]+\text { h.o.t. }
$$

Integrating both sides of the above equation from $-T$ to $T$, we arrive at

$$
\begin{aligned}
L(T)= & L(-T)+\int_{-T}^{T} U^{-1}(t) H_{\lambda}(\gamma(t), 0,0) \lambda d t \\
& +\int_{-T}^{T} U^{-1}(t) H_{\mu}(\gamma(t), 0,0) \mu d t+\text { h.o.t. }
\end{aligned}
$$

Noticing that $(\Phi(t))^{\prime}=U^{-1}(t)$, then we have the map $P_{1}$ : $S_{1} \mapsto S_{0}$ as follows:

$$
l_{i 1}=l_{i 0}+\lambda M_{i \lambda}+\mu M_{i \mu}+\text { h.o.t., } \quad i=1,3,4,
$$

where

$$
\begin{aligned}
& M_{i \lambda}=\int_{-T}^{T} \phi_{i}^{\prime} H_{\lambda}(\gamma(t), 0,0) d t=\int_{-\infty}^{\infty} \phi_{i}^{\prime} H_{\lambda}(\gamma(t), 0,0) d t, \\
& M_{i \mu}=\int_{-T}^{T} \phi_{i}^{\prime} H_{\mu}(\gamma(t), 0,0) d t=\int_{-\infty}^{\infty} \phi_{i}^{\prime} H_{\mu}(\gamma(t), 0,0) d t .
\end{aligned}
$$

(2) Establishment of the map $P_{0}: S_{0} \mapsto S_{1}$.

Let $\tau$ be the flying time from $q_{0}$ to $q_{1}$, and set $s=e^{-\rho \tau}$ (where $\rho=\min \left\{\rho_{1}(\alpha), \lambda_{1}(\alpha)\right\}$ ); utilizing the approximate solutions of system (5), it is easily to obtain the expression of $P_{0}: S_{0} \mapsto S_{1}$ :

$$
\begin{aligned}
& x_{0} \approx \frac{x_{1}}{\sqrt{h(s)}}, \quad y_{11} \approx s^{\rho_{1}(\alpha) / \rho} y_{10}, \\
& u_{0} \approx s^{\lambda_{1}(\alpha) / \rho} u_{1}, \quad y_{21} \approx s^{\rho_{2}(\alpha) / \rho} y_{20},
\end{aligned}
$$

where

$$
h(s)= \begin{cases}s^{2 \lambda_{p} / \rho}+\lambda_{p}^{-1}\left(x_{1}\right)^{2}\left(1-s^{2 \lambda_{p} / \rho}\right), & \lambda_{p} \neq 0, \\ 1-2(\rho)^{-1}\left(x_{1}\right)^{2} \ln s, & \lambda_{p}=0,\end{cases}
$$

and the higher order terms are neglected.

Remark 2. Figure 1 tells us that $x_{0} \approx x_{1} / \sqrt{h(s)}$ holds only when $x_{0} \geq \sqrt{\lambda_{p}}$, for $x_{0} \in\left[-\sqrt{\lambda_{p}}, \sqrt{\lambda_{p}}\right)$, the orbits near $O$ will go into $O^{0}$, and we may set $s=0$ in this case. While for $x_{0}<-\sqrt{\lambda_{p}}$, the orbits near $O$ will keep away from $O^{-}$. 
(3) Establishment of the map $P: S_{0} \mapsto S_{0}$.

Composing the maps $P_{0}$ and $P_{1}$, then $P=P_{1} \circ P_{0}: S_{0} \mapsto S_{0}$ can be expressed as

$$
\begin{aligned}
l_{11}= & \left(u_{12}\right)^{-1} s^{\rho_{1} / \rho} \delta-u_{42}\left(u_{12} u_{44}\right)^{-1} s^{\rho_{2} / \rho} y_{20} \\
& +\lambda M_{1 \lambda}+\mu M_{1 \mu}+\text { h.o.t., } \\
l_{31}= & x_{1}-u_{11}\left(u_{12}\right)^{-1} s^{\rho_{1} / \rho} \delta \\
& +\left[u_{11}\left(u_{12}\right)^{-1} u_{42}-u_{41}\right]\left(u_{44}\right)^{-1} s^{\rho_{2} / \rho} y_{20} \\
& +\lambda M_{3 \lambda}+\mu M_{3 \mu}+\text { h.o.t., } \\
l_{41}= & \left(u_{44}\right)^{-1} s^{\rho_{2} / \rho} y_{20}+\lambda M_{4 \lambda}+\mu M_{4 \mu}+\text { h.o.t.. }
\end{aligned}
$$

(4) Establishment of the successor function.

The successor function is given by $G\left(s, x_{1}, y_{20}\right)=(P-$ I) $\left(l_{10}, l_{30}, l_{40}\right)$ :

$$
\begin{aligned}
G_{1}= & \left(u_{12}\right)^{-1} s^{\rho_{1} / \rho} \delta-x_{0}+u_{31}\left(u_{33}\right)^{-1} s^{\lambda_{1} / \rho} \delta \\
& +\lambda M_{1 \lambda}+\mu M_{1 \mu}+\text { h.o.t., } \\
G_{3}= & x_{1}-u_{11}\left(u_{12}\right)^{-1} s^{\rho_{1} / \rho} \delta-\left(u_{33}\right)^{-1} s^{\lambda_{1} / \rho} \delta \\
& +\lambda M_{3 \lambda}+\mu M_{3 \mu}+\text { h.o.t., } \\
G_{4}= & {\left[\left(u_{44}\right)^{-1} s^{\rho_{2} / \rho}-1\right] y_{20}+\delta_{y} } \\
& +u_{14} x_{0}-\left(u_{14} u_{31}-u_{34}\right)\left(u_{33}\right)^{-1} s^{\rho_{1} / \rho} \delta \\
& +\lambda M_{4 \lambda}+\mu M_{4 \mu}+\text { h.o.t., }
\end{aligned}
$$

where $x_{0}$ is defined as (20).

\section{The Main Results}

We will discuss the homoclinic bifurcation accompanied by pitchfork bifurcation using the successor function achieved in Section 3.

It is obvious that system (1) has a homoclinic orbit or heteroclinic orbit (resp., periodic orbit) if and only if the equation

$$
\left(G_{1}, G_{3}, G_{4}\right)=0
$$

has a solution satisfying $s=0$ (resp., $s>0$ ).

According to the implicit function theorem, we know that the equation $G_{4}=0$ has a unique solution $y_{20}=y\left(s, x_{1}\right)$ for $\lambda$, $\mu$ sufficiently small, substituting it into $\left(G_{1}, G_{2}\right)=0$; then we obtain

$$
\begin{gathered}
\left(u_{12}\right)^{-1} s^{\rho_{1} / \rho} \delta-x_{0}+u_{31}\left(u_{33}\right)^{-1} s^{\lambda_{1} / \rho} \delta \\
+\lambda M_{1 \lambda}+\mu M_{1 \mu}+\text { h.o.t. }=0, \\
x_{1}-u_{11}\left(u_{12}\right)^{-1} s^{\rho_{1} / \rho} \delta-\left(u_{33}\right)^{-1} s^{\lambda_{1} / \rho} \delta \\
+\lambda M_{3 \lambda}+\mu M_{3 \mu}+\text { h.o.t. }=0 .
\end{gathered}
$$

Equation (25) is called the bifurcation equation.

Firstly, we consider homoclinic bifurcation with $\lambda=0$; that is, the pitchfork bifurcation does not happen. Based on
(20) and (21), we know that $x_{0} \approx x_{1} / \sqrt{1-2(\rho)^{-1}\left(x_{1}\right)^{2} \ln s}$ for $\lambda=0$; (25) then becomes

$$
\begin{aligned}
& \left(u_{12}\right)^{-1} s^{\rho_{1} / \rho} \delta-\frac{x_{1}}{\sqrt{1-2(\rho)^{-1}\left(x_{1}\right)^{2} \ln s}} \\
& +u_{31}\left(u_{33}\right)^{-1} s^{\lambda_{1} / \rho} \delta+\mu M_{1 \mu}+\text { h.o.t. }=0, \\
& x_{1}-u_{11}\left(u_{12}\right)^{-1} s^{\rho_{1} / \rho} \delta-\left(u_{33}\right)^{-1} s^{\lambda_{1} / \rho} \delta+\mu M_{3 \mu}+\text { h.o.t. }=0 .
\end{aligned}
$$

Note that $\rho=\min \left\{\lambda_{1}, \rho_{1}\right\}$; if $\rho_{1}<\lambda_{1}$ (resp., $\rho_{1}>\lambda_{1}$ ), then we have $s^{\rho_{1} / \rho}=s$ (resp., $\left.s^{\lambda_{1} / \rho}=s\right)$, and $s^{\lambda_{1} / \rho}=o(s)$ (resp., $s^{\rho_{1} / \rho}=$ $o(s)$ ), omitting the higher order term of $s$; it then follows that for $\rho_{1}<\lambda_{1}$ (for the case $\rho_{1}>\lambda_{1}$, we may discuss similarly), (26) turns to

$$
\begin{gathered}
\left(u_{12}\right)^{-1} s \delta-\frac{x_{1}}{\sqrt{1-2(\rho)^{-1}\left(x_{1}\right)^{2} \ln s}}+\mu M_{1 \mu}+\text { h.o.t. }=0, \\
x_{1}-u_{11}\left(u_{12}\right)^{-1} s \delta+\mu M_{3 \mu}+\text { h.o.t. }=0 .
\end{gathered}
$$

By way of the implicit function theorem, we know that if $\operatorname{rank}\left(M_{1 \mu}, M_{3 \mu}\right)=2$, there exists a $C^{r-2}$ function $\tilde{\mu}=$ $\psi\left(\mu^{*}, s, x_{1}\right)$ such that for $0 \leq s \ll 1$ (27) always holds. So we may obtain the following result.

Theorem 3. Suppose the conditions $\left(H_{1}\right)-\left(H_{3}\right)$ hold, $\lambda=0$ and $\operatorname{rank}\left(M_{1 \mu}, M_{3 \mu}\right)=2$; then

(i) as $\mu \neq \psi\left(\mu^{*}, s, x_{1}\right)$ for $0<s \ll 1, \mu$ small enough and fixed $\left|x_{1}\right| \ll 1$, system (1) has not any periodic orbit near $\Gamma$;

(ii) as $\mu=\psi\left(\mu^{*}, s, x_{1}\right)$ for $0<s \ll 1, \mu$ small enough and fixed $\left|x_{1}\right| \ll 1$, system (1) has a unique periodic orbit near $\Gamma$;

(iii) as $\mu=\psi\left(\mu^{*}, 0, x_{1}\right)$ for $\mu$ small enough and fixed $\left|x_{1}\right| \ll$ 1 , system (1) has a unique homoclinic orbit near $\Gamma$.

Remark 4. As we know that the homoclinic orbit is nongeneric, so the homoclinic orbit obtained in Theorem 3 (iii) comes from $O$ along the unstable manifold, while the orbit may come from the equilibrium $O$ along the weak unstable manifold (see Figure 2(b)).

Next, we consider the case $\lambda>0$; the origin undergoes a pitchfork bifurcation in this case; namely, there are three equilibria $\mathrm{O}^{+}, \mathrm{O}^{0}$, and $\mathrm{O}^{-}$bifurcated from the origin $\mathrm{O}$. And there always exist two straight segment orbits, one is heteroclinic to $\mathrm{O}^{+}$and $\mathrm{O}^{0}$ and the other is heteroclinic to $\mathrm{O}^{-}$ and $O^{0}$; their lengths are $\sqrt{\lambda_{p}}$, and we denote the heteroclinic orbits by $\Gamma^{*}$ and $\Gamma_{*}$, respectively. On the other hand, based on the definition of $P_{0}$, we will consider the bifurcations with three cases: for $x_{0} \geq \sqrt{\lambda_{p}}, x_{0} \in\left[-\sqrt{\lambda_{p}}, \sqrt{\lambda_{p}}\right)$ and $x_{0}<$ $-\sqrt{\lambda_{p}}$ 


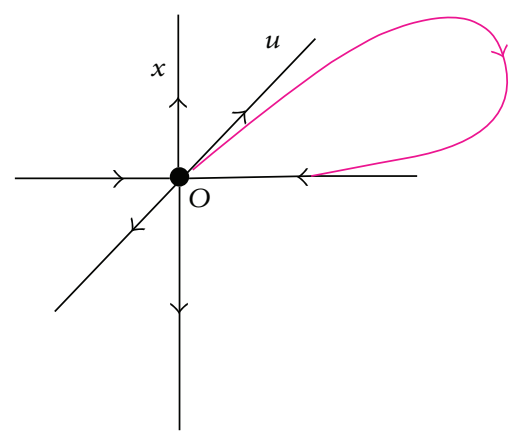

(a)

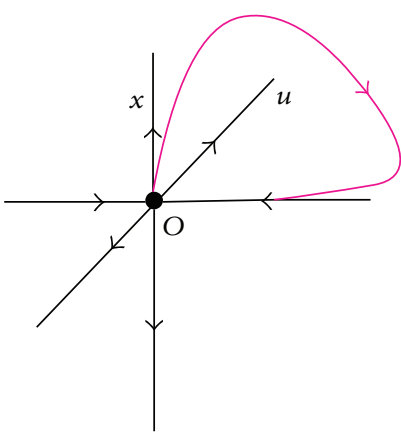

(b)

FIgURE 2: Homoclinic orbit with $O$.

First, we discuss the homoclinic bifurcation for $x_{0} \geq$ $\sqrt{\lambda_{p}}$. Based on (20), (25) turns to

$$
\begin{gathered}
\left(u_{12}\right)^{-1} s \delta-\frac{x_{1}}{\sqrt{s^{2 \lambda_{p} / \rho_{1}}+\lambda_{p}^{-1}\left(x_{1}\right)^{2}\left(1-s^{2 \lambda_{p} / \rho_{1}}\right)}} \\
+\lambda M_{1 \lambda}+\mu M_{1 \mu}+\text { h.o.t. }=0, \\
x_{1}-u_{11}\left(u_{12}\right)^{-1} s \delta+\lambda M_{3 \lambda}+\mu M_{3 \mu}+\text { h.o.t }=0,
\end{gathered}
$$

where we still consider the case $\rho_{1}<\lambda_{1}$, we may discuss the case for $\rho_{1} \geq \lambda_{1}$ similarly. Denote $r \triangleq s^{2 \lambda_{p} / \rho_{1}}$; then we get

$$
\begin{gathered}
\frac{x_{1}}{\sqrt{s^{2 \lambda_{p} / \rho_{1}}+\lambda_{p}^{-1}\left(x_{1}\right)^{2}\left(1-s^{\left.2 \lambda_{p} / \rho_{1}\right)}\right.}}=\frac{\sqrt{\lambda_{p}}}{\sqrt{1+\left(\left(x_{1}\right)^{-2} \lambda_{p}-1\right) r}} \\
=\sqrt{\lambda_{p}}+\frac{1}{2}\left(1-\left(x_{1}\right)^{-2} \lambda_{p}\right) r+\text { h.o.t.. }
\end{gathered}
$$

Equation (28) is then becomes

$$
\begin{aligned}
& \left(u_{12}\right)^{-1} r^{\rho_{1} / 2 \lambda_{p}} \delta-\sqrt{\lambda_{p}}-\frac{1}{2}\left(1-\left(x_{1}\right)^{-2} \lambda_{p}\right) r \\
& +\lambda M_{1 \lambda}+\mu M_{1 \mu}+\text { h.o.t. }=0 \\
& x_{1}-u_{11}\left(u_{12}\right)^{-1} r^{\rho_{1} / 2 \lambda_{p}} \delta+\lambda M_{3 \lambda}+\mu M_{3 \mu}+\text { h.o.t. }=0 .
\end{aligned}
$$

If $M_{1 \mu} \neq 0, M_{3 \mu} \neq 0,(30)$ gives a solution $\mu=\mu\left(r, x_{1}, \lambda\right)$ by virtue of implicit function theorem. And for fixed $\left|x_{1}\right| \ll$ 1 and the first equation of (30) we obtain $\mu^{*}(r, \lambda)=$ $\left(1 / M_{1 \mu}\right)\left[\sqrt{\lambda_{p}}+(1 / 2)\left(1-\left(x_{1}\right)^{-2} \lambda_{p}\right) r-\lambda M_{1 \lambda}-\left(u_{12}\right)^{-1} r^{\rho_{1} / 2 \lambda_{p}} \delta\right]+$ h.o.t., differentiating $\mu^{*}(r, \lambda)$ with respect to $r$, we achieve

$$
\frac{\partial \mu^{*}(r, \lambda)}{\partial r} \approx \frac{1}{2 M_{1 \mu}}\left(1-\left(x_{1}\right)^{-2} \lambda_{p}\right),
$$

notice that the $x_{0} \geq \sqrt{\lambda_{p}}$, according to the relation of $x_{1}$ and $x_{0}$ in (20) and (21), we may see that $1-\left(x_{1}\right)^{-2} \lambda_{p}>0$; then the above equations explore that $\left(\partial \mu^{*}(r, \lambda)\right) / r>0$ (resp., $\left.\partial \mu^{*}(r, \lambda) / r<0\right)$ as $M_{1 \mu}>0$ (resp., $\left.M_{1 \mu}<0\right)$, which implies that $\mu^{*}(r, \lambda)$ is monotonic with respect to $r$ when $M_{1 \mu} \neq 0$. Moreover,

$$
\begin{array}{r}
\mu^{*}(r, \lambda) \longrightarrow \mu^{*}(0, \lambda)=\frac{1}{M_{1 \mu}}\left[\sqrt{\lambda_{p}}-\lambda M_{1 \lambda}\right]+\text { h.o.t. } \\
\text { as } r \longrightarrow 0 .
\end{array}
$$

Therefore, we achieve the following conclusion.

Theorem 5. Let the hypotheses $\left(H_{1}\right)-\left(H_{3}\right)$ hold, $M_{1 \mu} \neq 0$, $M_{3 \mu} \neq 0$ and $0<\lambda \ll 1$; then in addition to the heteroclinic orbits $\Gamma^{*}$ and $\Gamma_{*}$,

(i) system (1) has a unique homoclinic orbit with $\mathrm{O}^{+}$for $\mu=\mu^{*}(0, \lambda)$ for fixed $\left|x_{1}\right| \ll 1$;

(ii) there exists $\mu_{1}>0$ small enough such that when $M_{1 \mu}>$ $0, \mu^{*}(0, \lambda)<\mu<\mu_{1}$, or $M_{1 \mu}<0,-\mu_{1}<\mu<\mu^{*}(0, \lambda)$ system (1) has a unique 1-periodic orbit near $\Gamma$.

Next, we discuss the case $x_{0} \in\left[-\sqrt{\lambda_{p}}, \sqrt{\lambda_{p}}\right)$, as we know that the orbit will go into $O^{0}$ (we denote $s=0$ ) in this case. While for $x_{0}<-\sqrt{\lambda_{p}}$ the orbit will keep away from $\mathrm{O}^{-}$. However, the orbit that comes from the equilibrium will be decided by $x_{1}$. If $x_{1}>\sqrt{\lambda_{p}}$, then the orbit comes from $\mathrm{O}^{+}$; if $0<x_{1}<\sqrt{\lambda_{p}}$, then the orbit comes from $O^{0}$; for $-\sqrt{\lambda_{p}}<x_{1}<0$, then the orbit comes from $O^{-}$(see Figure 1 ). So we obtain the following result.

Theorem 6. Let the conditions $\left(H_{1}\right)-\left(H_{3}\right)$ be true, and $0<$ $\lambda \ll 1, M_{i \mu}^{1} \neq 0, i=1,3$. Then in addition to the heteroclinic orbits $\Gamma^{*}$ and $\Gamma_{*}$,

(i) there exists a region in the $(\lambda, \mu)$ space:

$$
\begin{aligned}
& \Sigma_{1}(\mu, \lambda) \\
&:=\left\{(\lambda, \mu):-\sqrt{\lambda_{p}} \leq \lambda M_{1 \lambda}^{1}+\mu M_{1 \mu}^{1}+\text { h.o.t. }<\sqrt{\lambda_{p}}\right. \\
&\left.\quad \lambda M_{3 \lambda}+\mu M_{3 \mu}+\text { h.o.t. }>\sqrt{\lambda_{p}}, 0<|\mu|, \lambda \ll 1\right\}
\end{aligned}
$$

such that system (1) has a heteroclinic orbit with $\mathrm{O}^{+}$and $O^{0}$ as $(\lambda, \mu) \in \Sigma_{1}(\mu, \lambda)$; see Figures $3(a)-3(d)$; 


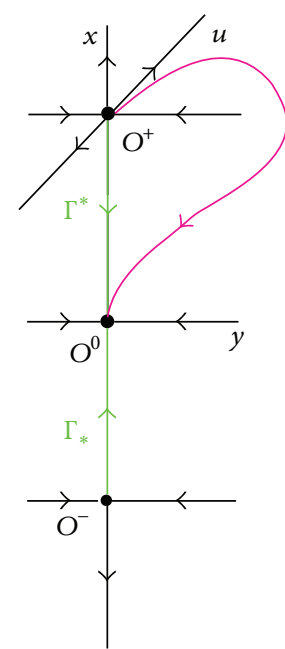

(a)

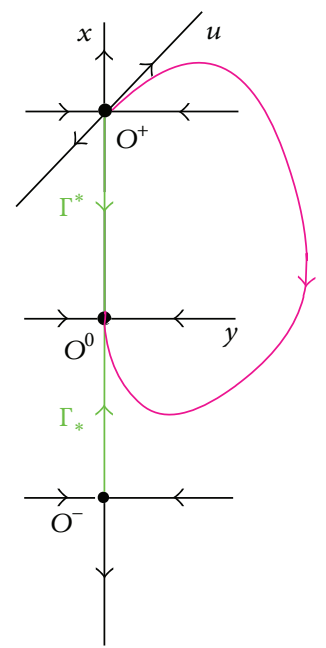

(b)

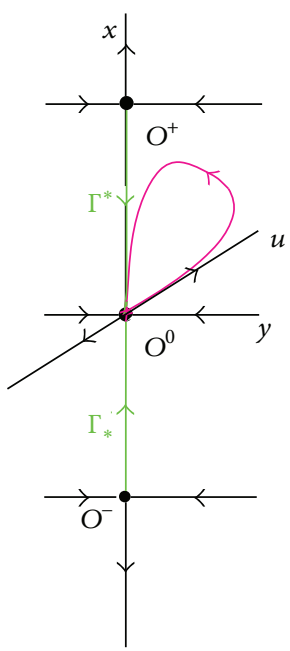

(f)

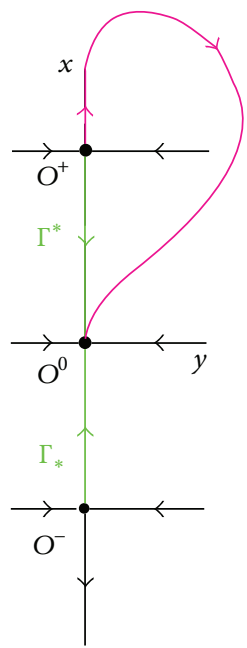

(c)

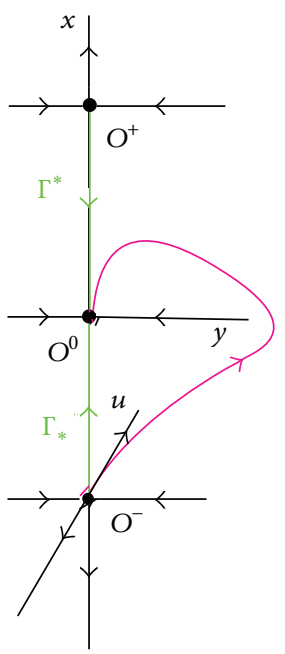

(g)

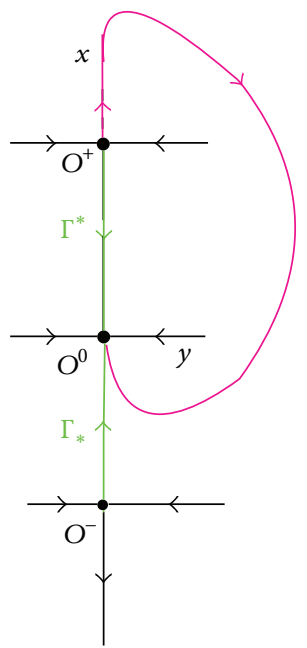

(d)

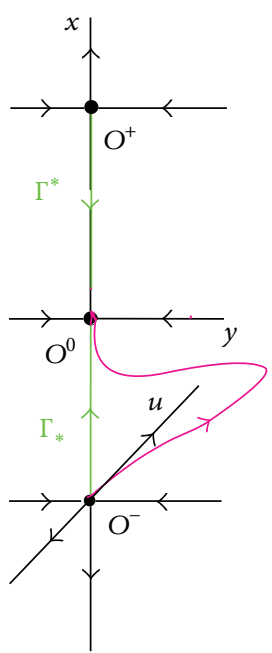

(h)

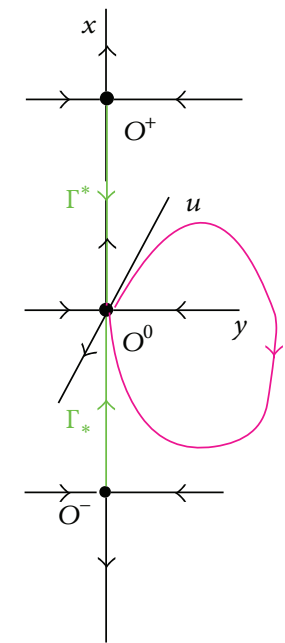

(e)

FIGURE 3: Homoclinic or heterclinic orbits accompanied by pitchfork bifurcation.

(ii) there exists a region in the $(\lambda, \mu)$ space:

$$
\begin{aligned}
& \Sigma_{2}(\mu, \lambda) \\
& :=\left\{(\lambda, \mu):-\sqrt{\lambda_{p}} \leq \lambda M_{1 \lambda}^{1}+\mu M_{1 \mu}^{1}+\text { h.o.t. }<\sqrt{\lambda_{p}},\right. \\
& \left.\quad 0<\lambda M_{3 \lambda}^{1}+\mu M_{3 \mu}^{1}+\text { h.o.t. }<\sqrt{\lambda_{p}}, 0<|\mu|, \lambda \ll 1\right\}
\end{aligned}
$$

such that system (1) has a homoclinic orbit connecting $O^{0}$ as $(\lambda, \mu) \in \Sigma_{2}(\mu, \lambda)$; see Figures $3(e)-3(f)$;

(iii) there exists a region in the $(\lambda, \mu)$ space:

$$
\begin{aligned}
\Sigma_{3}( & \mu, \lambda) \\
:= & \left\{(\lambda, \mu):-\sqrt{\lambda_{p}} \leq \lambda M_{1 \lambda}^{1}+\mu M_{1 \mu}^{1}+\text { h.o.t. }<\sqrt{\lambda_{p}},\right. \\
& \left.\quad-\sqrt{\lambda_{p}}<\lambda M_{3 \lambda}^{1}+\mu M_{3 \mu}^{1}+\text { h.o.t. }<0,0<|\mu|, \lambda \ll 1\right\}
\end{aligned}
$$

such that system (1) has a heteroclinic orbit connecting $O^{0}$ and $O^{-}$as $(\lambda, \mu) \in \Sigma_{3}(\mu, \lambda)$; see Figures $3(g)-3(h)$; (iv) there exists a region in the $(\lambda, \mu)$ space:

$$
\Sigma_{4}(\mu, \lambda):=\left\{(\lambda, \mu): \lambda M_{1 \lambda}^{1}+\mu M_{1 \mu}^{1}+\text { h.o.t. }<-\sqrt{\lambda_{p}}\right\}
$$

such that system (1) has no other heteroclinic orbit and no homoclinic orbit for $(\lambda, \mu) \in \Sigma_{4}(\mu, \lambda)$.

Remark 7. As we know from [3], the orbits in Figures 3(e)3(h) cannot be bifurcated from the generic homoclinic orbit, which exactly reveals the difference between the bifurcation of generic homoclinic orbit and the nongeneric one.

\section{Conflict of Interests}

The authors declare that there is no conflict of interests regarding the publication of this paper.

\section{Acknowledgments}

The authors are supported by National Natural Science Foundation of China (no. 11202192), the Fundamental Research 
Funds for the Central Universities (no. 2652012097), and the Beijing Higher Education Young Elite Teacher Project.

\section{References}

[1] D. M. Zhu, "The existence of nongeneric heteroclinic orbits accompanied by saddle-node bifurcation," Science in China, Series A, vol. 24, pp. 911-916, 1994.

[2] J. Klaus and J. Knobloch, "Bifurcation of homoclinic orbits to a saddle-center in reversible systems," International Journal of Bifurcation and Chaos, vol. 13, no. 9, pp. 2603-2622, 2003.

[3] X. Liu, X. Fu, and D. Zhu, "Homoclinic bifurcation with nonhyperbolic equilibria," Nonlinear Analysis: Theory, Methods \& Applications, vol. 66, no. 12, pp. 2931-2939, 2007.

[4] F. Geng and Y. Xu, "Bifurcations of heteroclinic loop accompanied by pitchfork bifurcation," Nonlinear Dynamics, vol. 70, no. 2, pp. 1645-1655, 2012.

[5] A. R. Champneys, "Codimension-one persistence beyond all orders of homoclinic orbits to singular saddle centres in reversible systems," Nonlinearity, vol. 14, no. 1, pp. 87-112, 2001.

[6] S.-N. Chow and X.-B. Lin, "Bifurcation of a homoclinic orbit with a saddle-node equilibrium," Differential and Integral Equations, vol. 3, no. 3, pp. 435-466, 1990.

[7] X. Liu, L. Shi, and D. Zhang, "Homoclinic flip bifurcation with a nonhyperbolic equilibrium," Nonlinear Dynamics, vol. 69, no. 12, pp. 655-665, 2012.

[8] T. Wagenknecht, "Two-heteroclinic orbits emerging in the reversible homoclinic pitchfork bifurcation," Nonlinearity, vol. 18, no. 2, pp. 527-542, 2005.

[9] S. Wiggins, Introduction to Applied Nonlinear Dynamical Systems and Chaos, vol. 2 of Texts in Applied Mathematics, Springer, New York, NY, USA, 1990. 


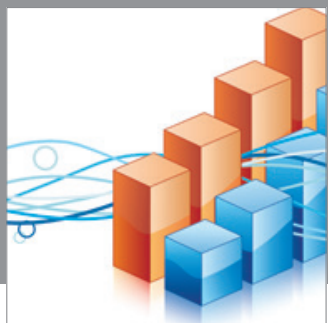

Advances in

Operations Research

mansans

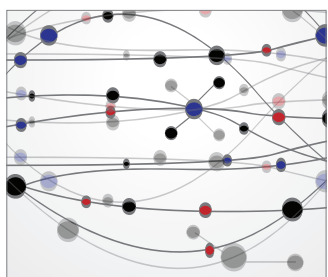

The Scientific World Journal
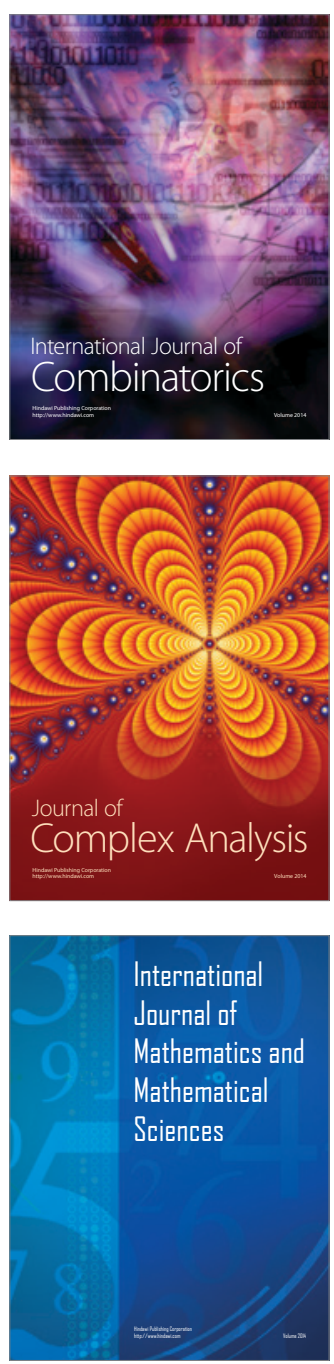
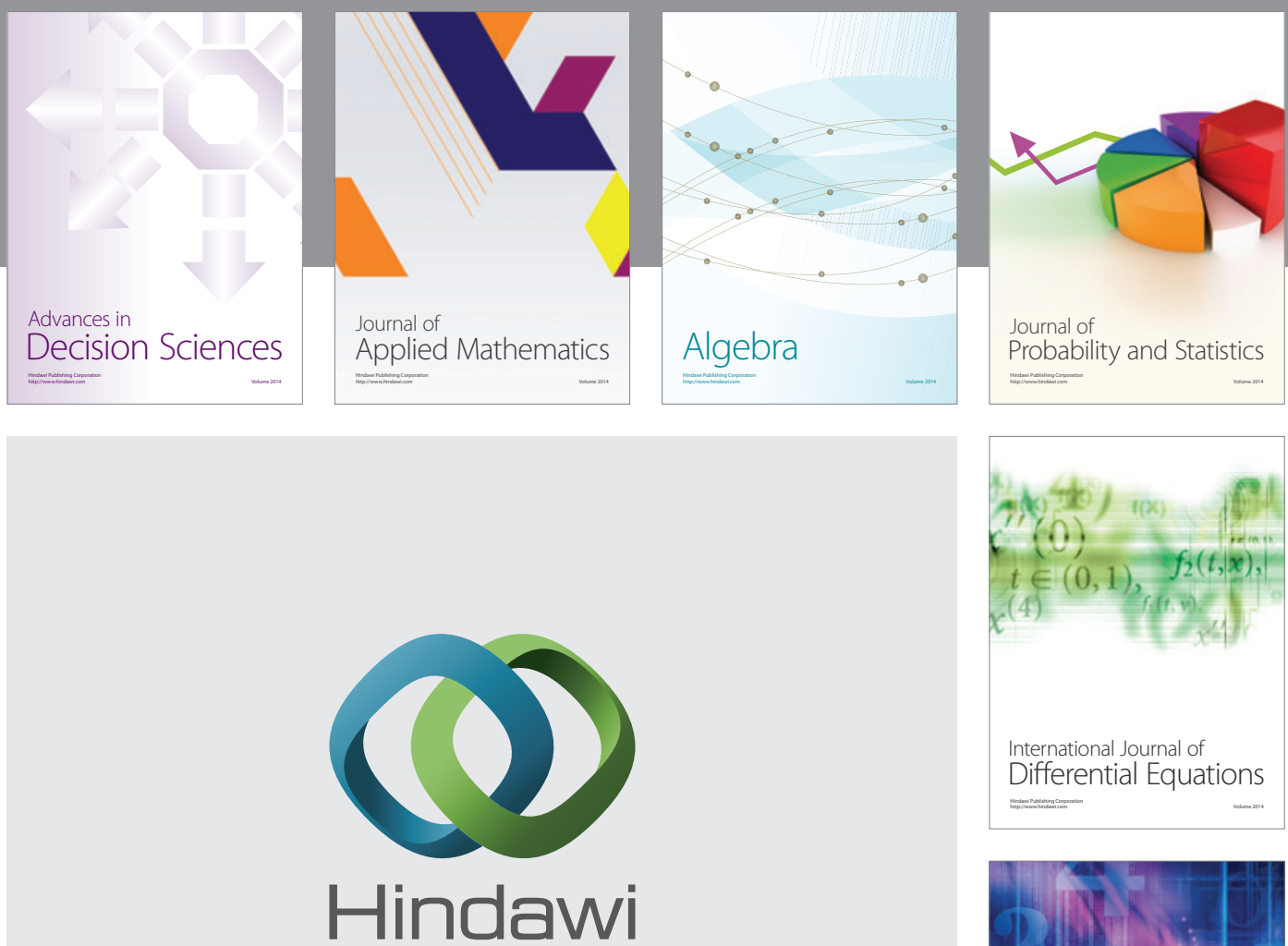

Submit your manuscripts at http://www.hindawi.com
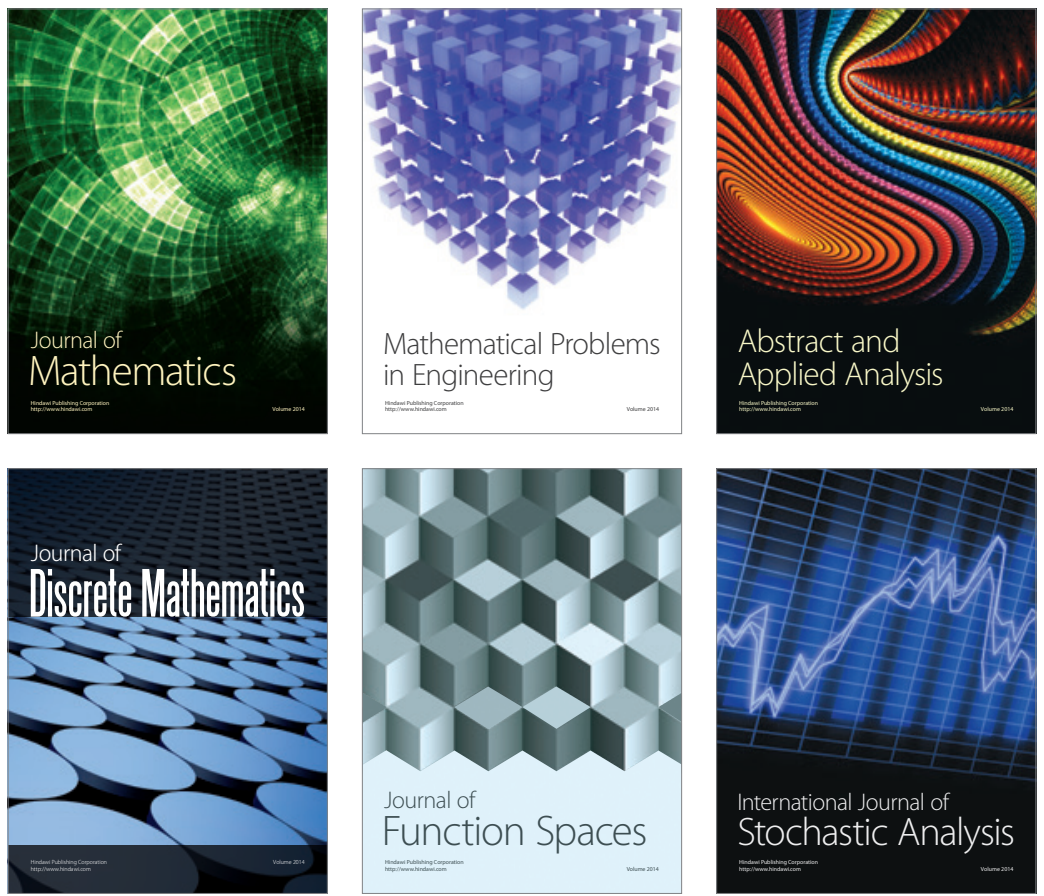

Journal of

Function Spaces

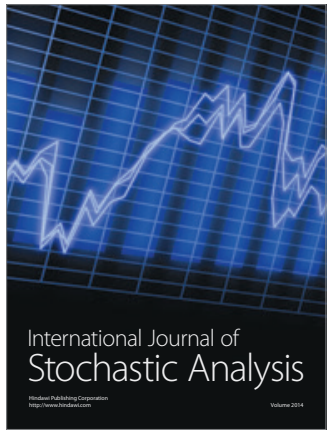

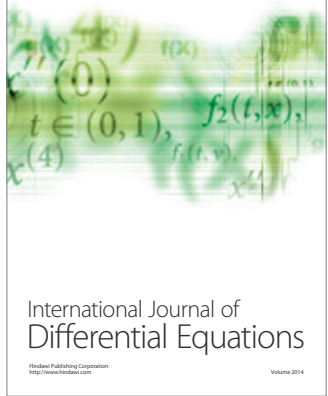
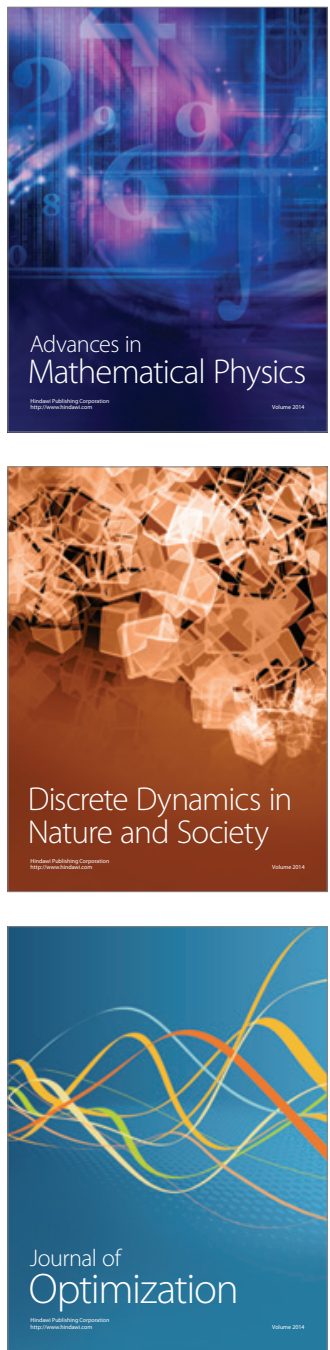